\title{
Los ODS como instrumento de aprendizaje: una experiencia multidisciplinar en los estudios universitarios
}

The SDGs as a learning tool: a multidisciplinary experience in university studies

Lucía Aparicio Chofré

Profa. Ayudante Doctora de Filosofía del Derecho

Departamento de Filosofía del Derecho y Política

Universidad de Valencia, España

E-mail: 1ucia.aparicio@uv.es

\section{Lupe Bohorques Marchori}

Profa. Adjunta en Grados de Ciencias Sociales y de la Salud

Universidad Europea de Valencia, España

E-mail: lupe.bohorques@ universidadeuropea.es

\section{Carla De Paredes Gallardo}

Profa. Adjunta en Grados de Ciencias Sociales y de la Salud

Universidad Europea de Valencia, España

E-mail: carla.deparedes@universidadeuropea.es

\section{Cristina Escamilla Robla}

Profa. Adjunta en Grados de Ciencias Sociales y de la Salud Universidad Europea de Valencia, España

E-mail: mariacristina.escamilla@universidadeuropea.es 


\title{
Elisa Giménez Fita
}

Profa. Adjunta en Grados de Ciencias Sociales y de la Salud

Universidad Europea de Valencia, España

E-mail: elisa.gimenez@universidadeuropea.es

\section{José María Quilez Moreno}

Prof. Titular de Derecho del Trabajo

Facultad de Ciencias Sociales

Universidad Europea de Valencia, España

E-mail: josemaria.quilez@universidadeuropea.es

\begin{abstract}
Resumen: La aprobación de la Agenda 2030 y de los Objetivos de Desarrollo Sostenible (ODS) representa una ambiciosa hoja de ruta para intentar dar respuesta a los desafíos globales y existenciales a los que se enfrenta a la humanidad. Bajo el lema de "No dejar a nadie a atrás" sus 17 objetivos comprenden cuestiones trascendentales que van desde la erradicación de la pobreza a la acción climática, pasando por el acceso a energías asequibles, fiables, sostenibles y modernas o el crecimiento económico sostenido, inclusivo y sostenible y terminando con la reducción de las desigualdades entre países o la promoción de sociedades pacíficas e inclusivas. En este contexto las Universidades, como el resto de los actores, están llamadas a desempeñar un papel crucial debido a su importante función como generadores y transmisores de conocimiento. Precisamente esta es la finalidad del presente proyecto de innovación educativa en el que nuevas metodologías de aprendizaje se utilizan para contribuir a este propósito. Se trata de una experiencia innovadora y multidisciplinar implantada durante varios cursos académicos en cuatro grados diferentes en la Universidad Europea de Valencia. Más de un centenar de estudiantes han tenido la oportunidad de participar en el proyecto obteniendo unos resultados altamente satisfactorios y una mejora evidente de sus conocimientos y competencias. Un proyecto, basado en alianzas y que posibilita que los estudiantes, en su condición de futuros líderes y gestores, no sólo conozcan y sean capaces de dar respuesta a estos importantes retos, sino que también adquieran una serie de competencias, las denominadas soft skills, que en la actualidad resultan imprescindibles.
\end{abstract}


Palabras clave: Objetivos de Desarrollo Sostenible, Aprendizaje por proyectos, Sostenibilidad, Transversalidad y Aprendizaje Servicio.

Abstract: The approval of the 2030 Agenda and the Sustainable Development Goals $(S D G)$ represents an ambitious roadmap which try to respond to the global and existential challenges that humanity faces. Under the slogan: "Leaving no one behind", its 17 Goals include transcendental issues ranging from poverty eradication to climate action, through access to affordable, reliable, sustainable and modern energy or sustained economic growth, inclusive and sustainable and ending with the reduction of inequalities between countries or the promotion of peaceful and inclusive societies. In this context, Universities, like the rest of the actors, are called to play a fundamental role due to their position as generators and transmitters of knowledge. This is precisely the purpose of this educational innovation project in which the SDGs are used as a learning tool. It is an innovative and multidisciplinary experience implemented during several academic years in four different degrees at the European University of Valencia. More than a hundred students have had the opportunity to participate in the project, obtaining highly satisfactory results and an evident improvement in their knowledge and skills. A project, in which different learning methodologies are combined, with the aim that students, as future leaders and managers, not only know and are able to respond to these important challenges, but also they acquire a series of competencies, the so-called soft skills, which are currently essential for a better quality education and more human.

Keywords: Sustainability Sustainable Development Goals, Project based Learning, transversality and Service Learning.

Sumario: 1. Introducción: Los ODS en el ámbito universitario. 2. Descripción proyecto: Los ODS como instrumento de aprendizaje. 3. Actividades prácticas. 4. Metodología. 5. Resultados del estudio de cambio en los estudiantes. 5.1. Diseño y análisis. 5.2. Instrumentos. 5.3. Descripción de la muestra. 5.4. Resultados de la evaluación de la eficacia. 5.4.1. Análisis comparativos sobre el nivel de conocimiento y la percepción positiva sobre la práctica de los ODS. 6. Conclusión. 7. Bibliografía. 


\section{Introducción: Los ODS en el ámbito universitario}

"Podemos utilizar la red global de universidades, su universidad, mi universidad, más de mil universidades en todo el mundo, para ser una red de soluciones activa para ayudar a los gobiernos, las empresas y la sociedad civil a trazar el camino hacia el éxito en el desarrollo sostenible, y también ser la incubadora para el rápido desarrollo de las tecnologías de desarrollo sostenible. Las universidades de todo el mundo deberían estar a la vanguardia a fin de ayudar a la sociedad a encontrar las soluciones técnicas para lograr estos objetivos" (SACHS, 2015: 61).

La Declaración de Naciones Unidas “Transformando nuestro mundo: la Agenda 2030 para el Desarrollo Sostenible", representa uno de los acuerdos más trascendentales a nivel humano y global de nuestra reciente historia. La resolución adoptada en la Cumbre de Naciones Unidas el 25 de septiembre de 2015 y firmada por todos sus estados miembros, supone un plan de acción a favor de las personas, el planeta y la prosperidad y aspira a situar al mundo y a sus sociedades en un camino hacia un futuro mejor (ONU, 2015).

Esta agenda es profundamente transformadora y supone un ambicioso proyecto que incluye una visión universal, unos principios comunes, una estrategia de ejecución y un marco de examen global en la que se implican no sólo los Estados miembros, sino también otros sectores como el empresarial, las ONGDs, las universidades tanto públicas como privadas y los ciudadanos de todos los países. La correcta o exitosa implementación de dichos objetivos conlleva un cambio sin precedentes al plantear una auténtica revolución en el terreno de las políticas públicas, empresariales y educativas.

Los 17 ODS apuntan a una misma dirección: convertir el mundo en un lugar más habitable y no dejar a nadie atrás. A partir de su lema "Transformar nuestro mundo", la Agenda gira alrededor de cinco ejes centrales que, a su vez, se relacionan con los ODS: Personas, Planeta, Prosperidad, Paz, y Alianzas (People, Planet, Prosperity, Peace and Partnership) (ONU, 2015).

Por otra parte, el objetivo número 4 de la Agenda 2030 se esfuerza en recalcar la importancia de promover una educación y aprendizaje inclusivo y de calidad a nivel mundial, y la meta 4.7 de este objetivo proclama la necesidad de promover el desarrollo 
sostenible mediante la educación y la promoción de una cultura de paz, ciudadanía mundial y la contribución de la cultura al desarrollo sostenible (DE LA ROSA et al., 2019).

Las instituciones universitarias tienen la oportunidad de poner a disposición de la Agenda 2030 sus recursos, sus estrategias y su espacio de influencia, al servicio de un desarrollo global sostenible no sólo respecto al ODS 4, sino al conjunto de los 17 objetivos planteados.

En esta labor, las universidades, como agentes sociales generadoras de conocimiento, que impactan de forma directa en las comunidades donde se ubican, están llamadas a desempeñar un papel de primer orden en la implementación de los ODS. El protagonismo de las universidades radica en su extensa variedad de actividades educativas y de aprendizaje (enseñanzas de grado y posgrado, prácticas profesionales, formación a mayores y a empresas, enseñanza a distancia, asociaciones estudiantiles, etc.) y en su capacidad para convertirse en un espacio que genera debate público y se interrelaciona con otras instituciones tanto públicas como privadas (CRUE, 2018).

Este intercambio con otras instituciones constituye una oportunidad, al tiempo que una exigencia, para responder a los desafíos del mundo. En este mismo contexto, el desarrollo de competencias relacionadas con los ODS es un reto para las instituciones de educación superior.

El Gobierno de España ha mantenido con las universidades una comunicación continua, a través de la Conferencia de Rectores de Universidades Españolas (CRUE) y se ha trabajado de forma activa en la puesta en marcha de la Agenda 2030 en los espacios que le son propios: formación, investigación, transferencia de conocimientos y la gestión universitaria. Este hecho quedó de manifiesto en la encuesta realizada por la CRUE el 24/04 de 2018, donde el 67\% de las 76 universidades manifestaba haber adoptado o estar trabajando en una estrategia específica con la Agenda 2030 (CRUE, 2018).

Desde marzo de 2019, la CRUE cuenta con una Comisión para la coordinación de acciones conjuntas que aseguran el cumplimiento de dichos objetivos, y a su vez, impulsan la concienciación con el entorno por parte de la comunidad universitaria para 
conseguir que la sociedad esté totalmente identificada con este espíritu de cambio (CRUE, 2019). De las numerosas iniciativas que se han impulsado, cabe destacar la Aportación de la CRUE reflejada en el Plan de Acción para la Implementación de la Agenda 2030, aprobado por el Consejo de Ministros el 29 de junio de 2018. Este Plan de Acción Estatal se ha concretado en numerosas acciones específicas universitarias, como son : la incorporación transversal de principios, valores y objetivos del desarrollo sostenible en la misión, políticas y actividades universitarias; un compromiso de inclusión en las competencias de desarrollo sostenible enfocado en los estudiantes, personal docente, investigador, administración y servicio; la generación y la transferencia de conocimiento; la capitalización de los espacios singulares para la puesta en marcha de proyectos innovadores; el fortalecimiento del vínculo de la universidad con otros agentes de la sociedad; la articulación de un debate público y abierto en torno al desarrollo sostenible; o el compromiso por parte de las universidades de registrar y realizar informes sobre impactos en términos de docencia, investigación y transferencia, alineándolos a cada uno de los ODS (Gobierno de España, 2018).

La mayoría de las universidades españolas han creado departamentos, cátedras o asignaturas que tienen una estrecha relación con la Responsabilidad Social y el Desarrollo Sostenible, donde se trabaja con rigor para que los alumnos/as y la propia Universidad sea un actor determinante del cambio (DE LA ROSA et al., 2019).

En este sentido, son varias las universidades españolas que han desarrollado distintos proyectos para la integración de los ODS, tanto en el ámbito académico como en la gestión de la propia Universidad. Estos proyectos tienen, su mayoría, un carácter específico como parte de una asignatura o un Máster y se enfocan, de forma preferente, en la sensibilización o concienciación del conocimiento sobre los ODS. Sin embargo, esta situación contrasta con los proyectos implementados a nivel internacional, especialmente en el mundo anglosajón, que destacan por un enfoque más holístico y multisectorial.

Aunque podríamos mencionar varios ejemplos de implementación y estudios que abordan la relación entre los ODS y la enseñanza universitaria, creemos que el conocimiento de los estudiantes sobre este tema aún no se ha introducido de forma satisfactoria, ni se ha creado una asignatura específica que contemple los ODS en profundidad. 
La Universidad Europea reconoce su alto compromiso social, ambiental, de transparencia y de responsabilidad corporativa y se suma al reto de incorporar los ODS a sus planes académicos en los distintos grados. Esta iniciativa supone un cambio que, sin duda, repercutirá de forma positiva en la formación de sus estudiantes como ciudadanos comprometidos con su entorno y los problemas globales (APARICIO et al., 2018).

Por ello, el proyecto de la Universidad Europea, llevado a cabo durante tres cursos consecutivos, se considera pionero en lograr la implementación de los ODS en sus aulas y un ejemplo de cómo se pueden introducir los ODS de forma transversal en cualquier grado universitario, asumiendo una percepción integral de los mismos. Nuestra propuesta incluye la formación en los ODS como un contenido esencial en los programas de las asignaturas, con un enfoque interdisciplinario, transdisciplinario y científico, que es posible alternar con los métodos de enseñanza tradicionales.

Además, creemos que es preciso implementar los ODS a través de la gestión y gobernanza de la Universidad, en su liderazgo e innovación, así como llevar a cabo actividades relacionadas con los ODS que demuestren el compromiso real de la Universidad con los 17 objetivos para contribuir al desarrollo y fomento de nuevas iniciativas.

La función de la enseñanza no debería quedar limitada a impartir sólo materias específicas, sino integrar otro tipo de herramientas que amplíen sus horizontes. Las universidades actúan como motor de cambio social porque, a través de la educación, se puede enseñar a los jóvenes líderes del futuro, nuevas maneras de estar en el mundo y de interactuar con el planeta. Los ODS necesitan a las universidades y las universidades a los ODS para formar a nuestros estudiantes como ciudadanos que se sienten parte del mundo en que viven y pueden contribuir a su mejora.

\section{Descripción proyecto: Los ODS como instrumento de aprendizaje}

Ante la importancia de los ODS en la actual sociedad, por parte de las universidades tenemos un compromiso social de hacer que nuestros alumnos universitarios conozcan los ODS. 
Desde la Universidad Europea de Valencia dentro de la Facultad de Ciencias Sociales y de la Salud, gracias a un proyecto de Investigación-Acción, aprobado en la convocatoria del año 2018, se ha tenido la oportunidad de implementar un proyecto basado en la contribución de la comunidad educativa en la Agenda 2030 como instrumento de aprendizaje.

En concreto este proyecto se implantó en los grados de Derecho, Relaciones Internacionales, Psicología, Criminología y Administración de Empresas, con la finalidad de elaborar un programa para la implementación de los ODS como herramienta educativa en el ámbito universitario.

Este proyecto se realizó durante 3 cursos académicos consecutivos y han participaron 6 docentes. Para la selección de las asignaturas del proyecto se tuvo en cuenta tanto el curso de impartición como el semestre, de tal forma que se pudiera implicar al máximo número de estudiantes y no hubiera una reiteración de contenidos.

De esta forma las asignaturas fueron: Derechos Humanos y Cooperación al Desarrollo y Ética, en el Grado de Relaciones Internacionales; en el Grado de Derecho en la asignatura de Derecho del Medio Ambiente y en Derecho Penal. En el Grado de Criminología en las asignaturas de Delincuencia y Control Social y Psicología del Testimonio; en el Doble Grado de Criminología y Psicología, las asignaturas de Psicología del Trabajo y de las Organizaciones y Psicología Social II; y en el Grado de Administración de Empresa, en Eficacia personal y profesional y Ética y Relaciones Laborales.

Al inicio del curso, en septiembre 2019, se les pasó a todos los alumnos de las distintas asignaturas un cuestionario previo para poder valorar cuáles eran los conocimientos iniciales. Las preguntas del cuestionario fueron generales respecto a los ODS, tales como: "tienes conocimiento sobre los ODS", "conoces el número de ODS y la Agenda 20/30", "conoces los principales retos a los que se enfrenta la humanidad en el próximo decenio", entre otras. Dicho cuestionario se les volvió a pasar, con las mismas preguntas, una vez terminado el proyecto al finalizar el semestre.

Una vez realizado el cuestionario previo, y teniendo en cuenta que sus conocimientos sobre la Agenda 2030 eran escasos, se explicó en el aula, con el apoyo de una presentación 
común elaborada por los docentes, cuáles eran los 17 ODS y la finalidad de cada uno de ellos para que pudieran tener un conocimiento uniforme y general sobre los ODS y poder empezar a trabajar todos con un mínimo de información.

Para trabajar, utilizando la metodología de la clase invertida, se puso a disposición de los alumnos distintos materiales como el Folleto sobre los ODS de Naciones Unidas, el Informe Un millón de voces el mundo que queremos, la Resolución aprobada por la Asamblea General de Naciones Unidas el 25 de septiembre de 2015, la Guía de los vagos para salvar el mundo, Naciones Unidas, Inspiring initiatives, partnerships and action to drive SDG implementation, Asamblea Naciones Unidas, y, por último, los MOOC ODS en la Agenda 2030 de las Naciones Unidas: Retos de los ODS.

Posteriormente, cada uno de los alumnos de las distintas asignaturas implicadas en el proyecto debía elegir un ODS entre los 17, para realizar una investigación en profundidad, siguiendo unas directrices previamente marcadas. De manera específica, en cada asignatura se diseñaron una serie de contenidos y prácticas determinados adaptados a la materia relacionados con los ODS.

Entre tanto se llevaron a cabo de manera trasversal y conjunta varias conferencias y actividades, que se detallarán en el siguiente apartado, en la misma Universidad y fuera de ella, y en colaboración con distintas entidades con la finalidad de que fueran interiorizando el sentido de los ODS.

Al finalizar el proyecto, la mayoría de los estudiantes manifestaron un elevado grado de satisfacción con sus prácticas debido al aprendizaje que les había reportado y un mayor compromiso con la Agenda 2030.

\section{Actividades prácticas}

Desde la Educación Superior, y en varios grados pertenecientes a los departamentos de Ciencias Sociales y Ciencias de la Salud, hemos contribuido proponiendo una serie de prácticas que puedan dar respuesta tanto a las necesidades sociales como ambientales que ambiciona el proyecto, a la vez que ayudamos a sensibilizar y tomar conciencia de nuestra responsabilidad social como ciudadanos y como institución. 
Las actividades planteadas desde la Universidad Europea de Valencia permiten, por tanto, implicar a los estudiantes en la sociedad, ilustrando la situación actual que están viviendo, aplicando los conocimientos teóricos de una forma práctica y profesional y conectándolos con la propia actualidad social y empresarial.

Estas actividades, perfectamente integradas en los planes de estudios, proporcionan esa parte práctica que tanto reclaman los estudiantes. Comprometidos con una responsabilidad social, permiten acercarlos a la realidad y ver cómo sus esfuerzos tienen una repercusión que les permite ir creando una identidad profesional desde la Universidad.

Además de dotar de conocimientos, este tipo de acciones posibilitan el desarrollo de habilidades y competencias como la cooperación entre ellos, el trabajo en equipo, la resolución de problemas auténticos, la creatividad, el análisis y la planificación, el pensamiento crítico y hacer posibles proyectos reales que contribuyan a desarrollar su experiencia, su curiosidad, su responsabilidad social y su empoderamiento.

El proyecto se llevó a cabo durante 3 años consecutivos, desde 2018 hasta 2021 de manera transversal. Consistió en la implementación de una serie de prácticas que cubrieran, por una parte, necesidades reales de las empresas y, por otra, que estuvieran conectadas con los contenidos, objetivos y competencias propias de la asignatura, con un propósito común a todos ellos: el compromiso, el fomento y la promoción de los ODS.

Se desarrollaron varios proyectos colaborativos con aplicación práctica, tanto nacionales como internacionales, que demostraron el compromiso del sector universitario con la Agenda 2030 y promovieron la participación activa de más de 100 estudiantes. Entre ellos podemos mencionar:

-La difusión de los ODS a través de las TIC's: algunas de las actividades llevadas a cabo utilizaban los recursos de las nuevas tecnologías para fomentar un conocimiento a gran escala de lo que son los ODS, su repercusión y alentaban la participación activa del público que, tras el visionado de las imágenes, ya era conocedor del proyecto y de la importancia de las pequeñas contribuciones. 
-Formación en distintas organizaciones, colegios, empresas, asociaciones: a través de diferentes versiones como charlas, exposiciones, conferencias, talleres experienciales, etc. Se proporcionó un aprendizaje de calidad hacia diferentes colectivos que abarcaban desde niños pequeños hasta directivos o personas jubiladas. Cambiando de registro para poder llegar e influir en todos los asistentes, nuestros alumnos pudieron, por una parte, experimentar lo que se siente al ser profesores y, por otra, desarrollar habilidades y competencias para lograr conseguir concienciar a su audiencia e influir en su actitud de cambio y de implicación.

Se diseñó un plan de acción para la introducción de determinados ODS dentro de una empresa ficticia, tomando como objetivo común el ODS 8 relativo al "Trabajo decente", y pudiendo seleccionar otros 3 adicionales de entre los 16 restantes que mejor se adaptasen a las características de la empresa seleccionada por los alumnos. De este modo, el alumnado pudo reflexionar acerca de dos cuestiones fundamentales: por un lado, el trabajo decente y, por el otro, inexorablemente unido al mismo, la salud laboral, elemento fundamental para lograr el bienestar personal y familiar en la esfera de las llamadas economías "avanzadas", donde las empresas han de cumplir con todos los estándares de calidad y de cumplimiento de la normativa laboral, especialmente la relacionada con la prevención de riesgos laborales.

Otras iniciativas relacionadas con la práctica en empresas reales fueron el diseño de acciones concretas como, acciones de voluntariado (con la contribución de un porcentaje de ventas a organizaciones benéficas), fomento de la educación digital y de las políticas efectivas de conciliación de la vida laboral y familiar, la adaptación de las instalaciones y sistemas de producción con tecnologías que favorecieran el consumo sostenible, empleo de energías renovables y cursos para los empleados con tales fines, la compra de productos de proveedores concienciados con el medio ambiente, o la participación en campañas de pesca sostenible y el uso de envases certificados y que respetaran el medio ambiente.

Cooperación con ONG, bancos de alimentos y de juguetes, compaginando la creatividad y la motivación por realizar un buen trabajo, y donde nuestros alumnos idearon varias formas de cooperar con diferentes organizaciones nacionales e internacionales. Algunas 
de las actividades consistieron en la colaboración con proyectos ya organizados, en los que eran una ayuda más que podía ser emparejada con el desarrollo de algunos de los ODS (educación para todos, fin de la pobreza, ciudades y comunidades sostenibles, etc.). Otros más ambiciosos, crearon una nueva forma de conseguirlo sin una base previa, como la creación de una red de distribución de juguetes en la época navideña, en la que se consiguió llevar juguetes reciclados españoles a niños colombianos.

Recogida de residuos en playas y bosques. Otras actividades promovieron campañas de recogida de residuos, reclutando voluntarios de la Universidad, y consiguiendo recoger varias toneladas de residuos que fueron luego transportados a puntos verdes de la localidad.

Reforestación. Se promovieron varias jornadas de voluntariado ambiental en las que se plantaron especies autóctonas en distintas zonas de la comunidad. Los alumnos también crearon carteles y colaboraron en la difusión de la actividad por medio de las redes sociales.

Actividades de reciclaje. Otras iniciativas consistieron en la promoción del reciclaje a distintos niveles, desde el fomento de cambios domésticos hasta otros proyectos que incluían grupos y organizaciones en las que se procuró un cambio en las actividades de recogida de basura y su clasificación.

Concienciación a través del arte y de la cultura. Varios proyectos se sirvieron de la habilidad artística de los alumnos para llevar a cabo actividades que sirvieran para concienciar y recaudar fondos. Entre ellas, podemos destacar exposiciones de cuadros y diseño de camisetas. Los fondos recaudados de la venta de las camisetas se destinaron a diferentes ONG como ECOMAR, una entidad sin ánimo de lucro fundada en 1999 por Theresa Zabell, única mujer española ganadora de dos Oros Olímpicos. Su principal función es la limpieza de costas con la ayuda de niños, educando a los más jóvenes en la importancia de preservar la salud de nuestros mares y cuidar el litoral de los países.

Este tipo de actividades, en las que se ha empleado una metodología que combina tanto la Investigación-Acción, como el Aprendizaje Servicio, suponen un valor añadido que posibilita a nuestros estudiantes extrapolar los conocimientos teóricos a la práctica, 
potenciar sus habilidades tanto personales como profesionales, acercarlos a una realidad compleja, cooperando desde distintos niveles de la misma, e implicarlos activamente en su propio aprendizaje y en su labor como ciudadanos responsables.

También se llevaron a cabo otras actividades outdoor en las que los alumnos pudieron vivenciar, a través de visitas programadas, las iniciativas que realizan otras entidades y su relación con el medio ambiente, como la organizada en la Central Nuclear de Cofrentes en relación con el ODS 7. La del Observatorio del Cambio Climático, coincidente con la celebración del día mundial de limpieza del planeta (ODS13), la empresa Social Net grupo GLOBAL y la visita al Centro de Interpretación de la Albufera, en la que se puso de manifiesto la relación con los ODS 13,14 y 15.

Se propuso una exposición "Los ODS a través de las iniciativas sociales", diseñada por la Fundación Alianza por la Solidaridad que estuvo visible en nuestras instalaciones para sensibilizar a toda la comunidad educativa.

También se programaron una serie de actividades, coincidentes con el día 3 de diciembre, en las que se trabajaron aspectos relacionados con el trabajo decente y la diversidad de capacidades a través de un taller experiencial a cargo del Grupo Integra sobre la diversidad funcional, cultural e intelectual y un mercadillo solidario realizado por el centro ocupacional Virgen de la Esperanza.

La biblioteca de la UEV se sumó al proyecto con una campaña de recogida de libros y juguetes: Toys for Ghana y la venta de libros para la ONG: Volunteers to Kenya.

Coincidente con la celebración del día 8 de marzo se realizó una obra de teatro y un taller de micromachismos. Además de las jornadas de sensibilización de charlas de los propios alumnos en colegios e institutos sobre la violencia machista junto con la colaboración de la ONG Intered.

Además de este tipo de actividades, la Universidad propuso, a través de su claustro de profesores, una serie de charlas y conferencias en distintos, foros, congresos y jornadas que sirvieran para concienciar y difundir la Agenda 2030 y la necesidad de implicar a las instituciones en tal propósito. Entre ellas podemos destacar la conferencia realizada por 
la profesora Lucía Aparicio Chofré sobre los Derechos Humanos y ODS en la Universidad de Valencia. El curso sobre Violencia de género en la Universidad de Valencia a cargo de la profesora Carmen Bellido. La Conferencia por parte de la Fundación Vicente Ferrer sobre el ODS 17 y las alianzas que se pueden fomentar entre la comunidad universitaria y las ONG. La Conferencia Proyecta y Bioagradable sobre la implantación de los ODS en la Comunidad Valenciana por el GRUPO GLOBAL y la Universidad Europea de Valencia. Alianza por la Solidaridad impartieron una serie de conferencias, destacando la de Mujeres y ODS y la de Defensoras y Derechos Humanos en conflictos socioambientales en el sur global. Manos Unidas también quiso contribuir con su participación en este proyecto para fomentar la participación voluntaria de nuestros alumnos, a través de un desayuno solidario para presentar las iniciativas relacionadas con la Agenda 2030. Intermón Oxfam también contribuyó el día del comercio justo visualizando su relación con el ODS 12 mediante la venta de productos de comercio justo y la implicación de la cafetería del Campus. Además, los docentes comprometidos con este proyecto participaron como ponentes en diferentes congresos y jornadas como: la Jornada sobre Derechos Humanos y ODS en la Universidad de Valencia (2018); el Foro Internacional sobre la Evaluación de la Calidad de la Investigación y de la Educación Superior (FECIES), con el simposio titulado: "La introducción de los ODS en las aulas universitarias" (APARICIO et al., 2019), véase Figura 1 o el Congreso Internacional sobre Derechos Humanos organizado por la Fundación Mainel (Aparicio, L y De Paredes, C, 2018).

Figura 1. Portada del Simposio "La introducción de los ODS en las aulas universitarias" (APARICIO et al. 2019).

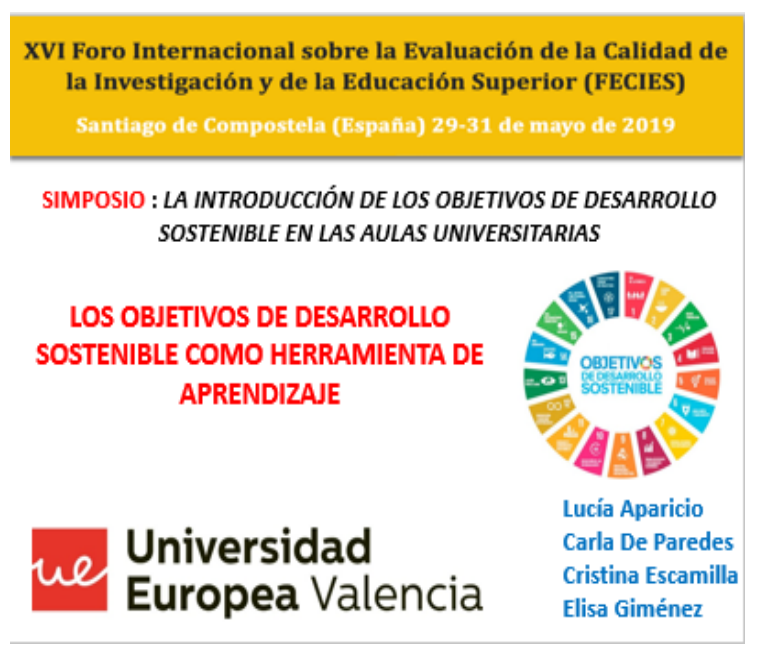


Asimismo, se celebró en la propia Universidad, el I Congreso sobre los ODS y la Agenda 2030, “QQué podemos aportar a la Sociedad en defensa del futuro de la Humanidad?”, véase Figura 2, cuyo objetivo principal fue presentar los trabajos y prácticas realizadas por los estudiantes participantes en el proyecto. Fue inaugurado por la Rectora y la Directora General de Cooperación Internacional para el Desarrollo de la Generalitat Valenciana.

Figura 2. Portada del folleto para la difusión del Congreso (Universidad Europea de Valencia, 2019)

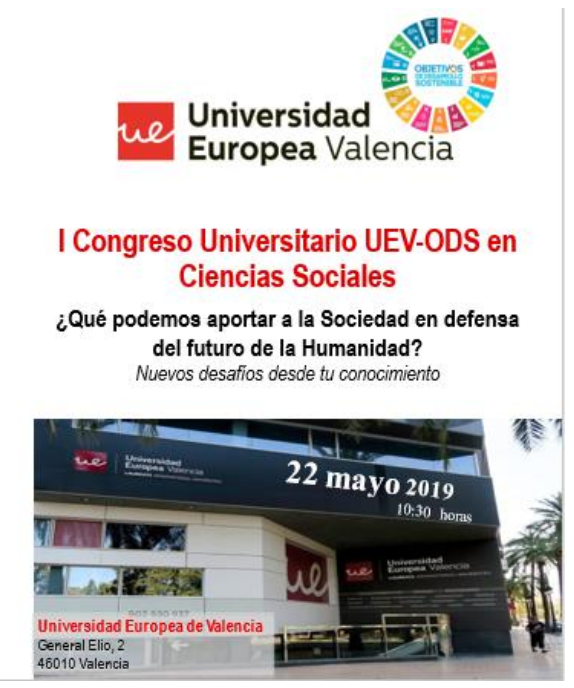

Todas las actividades llevadas a cabo en este proyecto se encuentran alineadas dentro de las directrices académicas y permiten que los estudiantes puedan promocionar sus habilidades personales y profesionales. Permiten que sean conscientes de la realidad que provoca un mundo en constante cambio, cada vez más comprometido con las situaciones sociales, y abierto a nuevas propuestas relacionadas con el fomento de la Responsabilidad Social Universitaria.

Desde la Universidad Europea de Valencia, hemos querido contribuir desarrollando diferentes iniciativas como una forma de aprendizaje basado en la experiencia y en las que se implican desde muy jóvenes con la Agenda 2030 y los ODS.

La acogida por parte de los estudiantes resultó muy buena coincidiendo en su gran mayoría en que este tipo de prácticas suponen un valor añadido que no hubiera sido 
posible alcanzar desde los sistemas de enseñanza más tradicionales. Permiten que su motivación e implicación sea más elevada y su visión menos sesgada y comprometida.

Alineados con todos los ODS, y especialmente con el ODS 17, en el que el fomento de las alianzas es crucial para poder conseguir todos estos propósitos conjuntos y conseguir desarrollar proyectos sociales y solidarios a gran escala, se crean sinergias y simbiosis que impulsan una estrategia global, ambiciosa y comprometida en sí misma. Poder seguir trabajando las alianzas entre ONG como en nuestro caso, Intered, Alianza por la Solidaridad, Fundación Vicente Ferrer, entre otras, nos siguen acercando a seguir cumpliendo el ODS 17.

\section{Metodología}

En cuanto a la metodología resulta oportuno realizar una doble distinción. En primer lugar, la metodología empleada para la implementación del proyecto en cada una de las asignaturas implicadas y las actividades transversales conjuntas realizadas; en segundo lugar, la propia metodología interna utilizada para el diseño, el desarrollo y la evaluación del proyecto.

Por lo que respecta a la primera, la relativa a la implementación del proyecto, ésta se caracteriza por su heterogeneidad y flexibilidad. De este modo, a fin de llevar a cabo la ejecución del proyecto se brindó una total flexibilidad a los docentes implicados para que adecuaran el proyecto a la metodología que consideraran más oportuna en atención a los contenidos de sus respectivas asignaturas y a los objetivos de aprendizaje de los diferentes grados.

Así, para el desarrollo del proyecto se han combinado las siguientes metodologías educativas: desde la tradicional clase magistral, a la flipped classroom, pasando por el aprendizaje basado en proyectos (Project based learning) y el aprendizaje servicio, continuando con el aprendizaje cooperativo y la simulación, y concluyendo con la evaluación por pares o la gamificación.

Algunos ejemplos concretos de la utilización de estas metodologías los encontramos en las siguientes actividades: 
- La clase magistral: en la mayoría de las asignaturas implicadas en el proyecto, tuvo una relevancia residual. Su empleo resultó necesario a fin de proporcionar a los estudiantes unos contenidos mínimos y homogéneos sobre los ODS. Para ello se elaboraron unos materiales conjuntos como una presentación en Power Point y una guía bibliográfica, que se distribuyeron entre todos los docentes participantes en el proyecto.

- La flipped classroom: se utilizó en la mayoría de las asignaturas al plantear una primera práctica introductoria común, en la que se les proporcionaba a los estudiantes una serie de materiales bibliográficos. También se empleó en otras asignaturas donde los estudiantes, a través de diferentes prácticas, tuvieron la oportunidad de trabajar de una forma invertida algunos ODS.

- $\quad$ El Aprendizaje por Proyectos, se introdujo en varias asignaturas en las que los estudiantes tuvieron que realizar proyectos concretos relacionados con los ODS.

- $\quad$ El Aprendizaje servicio se desarrolló en diferentes asignaturas donde, como se ha detallado en el apartado anterior, los estudiantes tuvieron la oportunidad de colaborar con diferentes entidades del tercer sector social, en materia social, medioambiente, arte y cultura, infancia, etc. Una actividad que resultó altamente gratificante, tanto para los estudiantes y profesores como para las entidades colaboradoras.

- El aprendizaje colaborativo se implementó, tanto con la realización de determinados proyectos en grupo o por equipos, como por la exposición en el aula de los proyectos y actividades desarrollados, en los que se fomentaba ese aprendizaje cooperativo. En algunos casos, tuvimos la sorpresa de comprobar que traspasó las paredes del aula y se extendió incluso a amigos y familiares. También contribuyeron a este aprendizaje las diversas actividades, como charlas, conferencias y exposiciones, que se organizaron en colaboración con diferentes entidades.

- La simulación se empleó principalmente con la organización del Congreso científico en el que se seleccionaron los mejores proyectos y trabajos realizados a fin de que se presentarán por parte de los estudiantes como comunicaciones. Posteriormente, los propios estudiantes votaron las que más les habían gustado y se les otorgó un premio. 
- La evaluación por pares se implementó tanto en el Congreso científico como para la evaluación de algunos de los proyectos. A tal fin se elaboró una rúbrica que los estudiantes debían cumplimentar tras asistir a las presentaciones orales en clase de dichos proyectos, y que luego el docente tenía en cuenta a la hora de la calificación final de la actividad.

- Gamificación: en algunas de estas actividades, tanto por los propios alumnos en sus presentaciones como por los docentes, se utilizaron herramientas como el Kahoot para evaluar los conocimientos adquiridos.

En segundo lugar, por lo que respecta a la propia metodología interna utilizada para el diseño, el desarrollo y la evaluación del proyecto, hay que destacar que se estableció un plan de acción que contemplaba la realización de una serie de reuniones mensuales para su correcto seguimiento.

Durante el transcurso de dichas reuniones, bajo la coordinación de la investigadora principal, se distribuyeron las diferentes tareas a realizar entre los integrantes del equipo, basándose en un sistema abierto, colaborativo y participativo, como la elaboración de materiales conjuntos, diseño de actividades, creación de cuestionarios de evaluación, análisis de resultados, contacto con entidades, y organización de actividades. Al margen de estas reuniones, el contacto y la implicación de todos los integrantes del proyecto fue constante y fluido, lo que facilitó su excelente desarrollo.

Como en cualquier proyecto, en momentos puntuales surgieron algunos problemas de coordinación logística -es conveniente recordar que no se contaba con ningún tipo de financiación-, pero gracias a la buena sintonía y disponibilidad de todos los participantes, se lograron superar y permitieron enriquecer y mejorar su implementación. Un proyecto que difícilmente habría salido adelante sin la confianza, el compromiso y el entusiasmo de los docentes implicados, ya que en muchas ocasiones suponía una carga de trabajo extra y un recorte de su tiempo personal para la dedicación al mismo. 


\section{Resultados del estudio de cambio en los estudiantes}

Uno de los objetivos de este proyecto era estudiar los cambios en cuanto a conocimientos y competencias de los estudiantes después de participar en el programa elaborado para implementar los ODS, y comprobar la eficacia de la metodología empleada.

\subsection{Diseño y análisis}

Se planteó un diseño longitudinal de medidas repetidas, antes y después, con una evaluación del cambio en un plazo medio de tiempo de seis meses, incluyendo dos ocasiones secuenciales por sujeto: una al inicio de la asignatura, coincidiendo con el inicio del semestre; y otra con la finalización de este. Se realizaron análisis estadísticos en concordancia con el tipo de diseño planteado para comprobar la magnitud del cambio en los estudiantes: contrastes de medias repetidas (prueba t de Student) para muestras relacionadas, previa comprobación de la normalidad (prueba Kolmogorov-Smirnov).

\subsection{Instrumentos}

Los instrumentos utilizados para la evaluación de la eficacia de la metodología sobre los ODS fueron dos cuestionarios idénticos, pre y post, elaborados para este estudio. Consta Constaban de 16 ítems: los diez primeros, medían el nivel de conocimiento de los estudiantes sobre los ODS; y los seis restantes, medían la percepción positiva de los alumnos sobre su participación en la práctica de los ODS, y la mejora en su nivel de competencias. Por último, se midió en el segundo cuestionario (post), la satisfacción, añadiendo una última pregunta con el fin de que valoraran su satisfacción con la introducción de la nueva herramienta educativa.

\subsection{Descripción de la muestra}

Se consiguió una muestra de 120 estudiantes, con una media de edad de 21 años, siendo la edad más alta la de 33 y la más baja de 17 años; un 51\% mujeres y un $49 \%$ hombres; y pertenecían el 19\% a la titulación de Derecho, el 18\% a Psicología, el 17\% a la Doble de Criminología con Psicología, un 21\% a ADE; y un 25\% a RRII. 


\subsection{Resultados de la evaluación de la eficacia}

En este apartado se detallan los resultados obtenidos sobre la evaluación de la eficacia de la metodología de los ODS, estructurados en tres apartados. El primero describe los análisis comparativos entre las dos medidas repetidas estudiadas, estableciendo las comparaciones de dos variables, por una parte, el conocimiento de los estudiantes acerca de los ODS y, y por otra, la percepción positiva sobre la participación en la práctica de los ODS. El segundo, analiza la satisfacción de los estudiantes con la metodología empleada en el proyecto.

5.4.1. Análisis comparativos sobre el nivel de conocimiento y la percepción positiva sobre la práctica de los ODS

Para conocer el nivel de conocimiento acerca de los ODS, se extrajo la puntuación para cada sujeto a partir de la suma de los 10 ítems formulados en el cuestionario y cuyas respuestas estaban medidas con puntaciones escala Likert, entre 1 a 5 puntos, cuando el 1 significaba "Totalmente en desacuerdo" y el 5 "Totalmente de acuerdo", de forma que la puntuación máxima son 50 puntos. Asimismo, se extrajo una puntuación sobre la percepción positiva, utilizando el mismo tipo de respuestas, pero con la suma de los 6 ítems planteados, de forma que la puntuación máxima era de 30 puntos. Dichas sumas se establecieron en dos momentos: antes y después de la intervención. En la Tabla 1 se reflejan los resultados.

Tabla 1. Medias, desviaciones típicas de dos ocasiones (antes y después), y contraste de 2 medias relacionadas sobre el nivel de conocimiento y la percepción positiva sobre la práctica de los ODS

\begin{tabular}{|l|c|c|c|c|c|}
\multicolumn{1}{c}{} & \multicolumn{3}{c}{$\begin{array}{c}\text { Antes } \\
(\mathbf{N}=\mathbf{1 2 0})\end{array}$} & \multicolumn{2}{c}{$\begin{array}{c}\text { Después } \\
(\mathbf{N = 1 2 0})\end{array}$} \\
\cline { 2 - 6 } & $\mathrm{M}$ & $\mathrm{DT}$ & $\mathrm{M}$ & $\mathrm{DT}$ & $t$ \\
\hline Nivel de conocimiento & 18.93 & 6.74 & 40.44 & 7.49 & $18.58^{* * *}$ \\
\hline Percepción positiva & 21.72 & 6.81 & 26.42 & 4.22 & $5.12 * * *$ \\
\hline
\end{tabular}

Nota. Abreviaturas: M: media; DT: desviación típica; $t=\mathrm{t}$ de Student; $* * *=\mathrm{p}<.001$.

Se observa un aumento de las medias en el segundo pase de los cuestionarios, y donde los estadísticos muestran en las dos pruebas un $\mathrm{p}$ valor <.001, por tanto, se concluye que el nivel de conocimiento acerca de los ODS y la percepción positiva sobre la participación 
en la práctica de los ODS, aumentan de forma estadísticamente significativa después de la introducción de la metodología en el aula. Es decir, que se produce un cambio significativo en el conocimiento y en la percepción positiva de los estudiantes.

A continuación, se plantea un análisis más detallado de las dos variables estudiadas, donde se muestran las comparaciones de cada ítem. En la Tabla 2, se puede observar las diferencias de las puntuaciones de todos los ítems relacionados con el conocimiento, observándose medias más altas en todos los ítems después de la intervención con la metodología empleada.

Tabla 2. Medias, desviaciones típicas de dos ocasiones (antes y después), y contraste de 2 medias relacionadas de los ítems que miden el nivel de conocimiento sobre los ODS

Ítems sobre contenidos sobre ODS

Antes Después

\begin{tabular}{|c|l|l|l|l}
\multicolumn{3}{|c}{$(\mathbf{N}=120)$} & \multicolumn{3}{c}{$(\mathbf{N}=120)$} \\
\hline $\mathrm{M}$ & $\mathrm{DT}$ & $\mathrm{M}$ & $\mathrm{DT}$ & $t$ \\
\hline 2.07 & 1.19 & 4.33 & .83 & $14.12^{* * *}$ \\
\hline 1.49 & 1.03 & 4.51 & .91 & $19.06 * * *$ \\
\hline 1.86 & 1.13 & 4.30 & .92 & $14.49 * * *$ \\
\hline 1.56 & .96 & 3.66 & 1.20 & $11.05 * * *$ \\
\hline 1.44 & .86 & 4.09 & 1.02 & $17.04 * * *$ \\
\hline 1.77 & .96 & 3.67 & 1.00 & $11.39 * * *$ \\
\hline 1.89 & 1.30 & 3.71 & 1.38 & $8.38 * * *$ \\
\hline 1.56 & .88 & 3.71 & 1.12 & $11.95 * * *$ \\
\hline 2.56 & 1.17 & 4.23 & .83 & $10.81 * * *$ \\
\hline 2.74 & 1.66 & 4.23 & .95 & $7.13 * * *$ \\
\hline
\end{tabular}

1. Tengo conocimientos sobre los ODS

2. Conozco el número de ODS

3. Sabría explicar brevemente en qué consisten los ODS

4. Tengo conocimientos previos sobre los Objetivos del Milenio

5. Tengo conocimientos sobre la Agenda 20/30

6. Tengo conocimientos sobre la Alianza Mundial

7. Tengo conocimiento sobre lo que es RSC

8. Tengo conocimiento sobre el Global Compact

9. Conozco los principales retos a los que se enfrenta la humanidad en el próximo decenio

10. Entiendo el concepto de resiliencia

Nota. Abreviaturas: M: media; DT: desviación típica; $t=\mathrm{t}$ de Student.

$$
*=\mathrm{p}<.05 ; * *=\mathrm{p}<.01 \mathrm{y} * * *=\mathrm{p}<.001 \text {. }
$$

Se observa que los p valor obtenidos en todos los ítems son <.001, concluyéndose que existen diferencias estadísticamente significativas en todos los ítems relacionados con el nivel de conocimiento acerca de los ODS.

En la Tabla 3 se reflejan las diferencias de las puntuaciones de todos los ítems relacionados con la percepción positiva de la participación en la práctica de los ODS, y donde también se observan medias más altas después de la intervención con la metodología empleada. 
Tabla 3. Medias, desviaciones típicas, en dos ocasiones (antes y después), y contraste de 2 medias de los ítems que estudian la percepción sobre los ODS

\begin{abstract}
Ítems sobre percepción sobre ODS
1. Comparado con el esfuerzo que supone, la participación en la práctica de los ODS pienso que puede resultar beneficiosa
\end{abstract}

\begin{tabular}{|l|l|l|l|l|l|}
\hline $\begin{array}{l}\text { 2. Me parece interesante participar en este tipo de } \\
\text { actividades }\end{array}$ & 3.51 & 1.25 & 4.46 & .75 & $5.62^{* * *}$ \\
\hline $\begin{array}{l}\text { 3. Participar en la práctica de los ODS creo puede aportar } \\
\text { valor educativo }\end{array}$ & 3.70 & 1.24 & 4.51 & .76 & $4.61^{* * *}$ \\
\hline $\begin{array}{l}\text { 4. Participar en la práctica de los ODS puede promover una } \\
\text { visión práctica y aplicada de conocimientos }\end{array}$ & 3.63 & 1.37 & 4.39 & .80 & $3.87^{* * *}$ \\
\hline $\begin{array}{l}\text { 5. En general, la práctica de los ODS puede servir para } \\
\text { trabajar y mejorar competencias }\end{array}$ & 3.63 & 1.35 & 4.34 & .89 & $3.87^{* * *}$ \\
\hline $\begin{array}{l}\text { 6. Consideras positiva la introducción de este tipo de } \\
\text { metodologías en el aula? }\end{array}$ & 3.81 & 1.24 & 4.37 & .85 & $3.51^{* * *}$ \\
\hline
\end{tabular}

\section{Nota. Abreviaturas: M: media; DT: desviación típica; $t=\mathrm{t}$ de Student.

$$
*=\mathrm{p}<.05 ; * *=\mathrm{p}<.01 \mathrm{y} * * *=\mathrm{p}<.001
$$

Los resultados son acordes a los análisis anteriores, observándose que los p valor obtenidos en todos los ítems son <.001, por lo que se concluye que existen diferencias estadísticamente significativas en todos los ítems relacionados con la percepción positiva de la práctica de los ODS en el aula, antes de la introducción de la metodología y después.

5.4.2. Análisis de la satisfacción de los estudiantes con la intervención de la metodología de empleada en la asignatura

A continuación, se presentan los resultados sobre la satisfacción de los estudiantes con la nueva metodología. Se evaluó mediante una única pregunta con respuesta escalar de 1 a 5 puntos. En la Tabla 4 se puede observar la media de dicha puntuación.

Tabla 4. Media y desviación típica de la satisfacción de los estudiantes con la metodología de los ODS

\begin{tabular}{|c|c|c|}
\hline & \multicolumn{2}{|c|}{$\begin{array}{l}\text { Estudiantes } \\
\qquad(\mathbf{N}=\mathbf{1 2 0})\end{array}$} \\
\hline & $\mathbf{M}$ & DT \\
\hline $\begin{array}{l}\text { 7. Estoy satisfecho con el planteamiento del trabajo de investigación sobre los } \\
\text { ODS }\end{array}$ & 4.47 & .79 \\
\hline
\end{tabular}


La media del grado de satisfacción es de 4.47, muy cercana al 5, por lo que se concluye que la satisfacción de los estudiantes con la nueva metodología de los ODS es muy alta.

\section{Conclusión}

De la realización del presente proyecto de innovación-acción se pueden extraer las siguientes conclusiones

Primera. A pesar de haber transcurrido más de un lustro desde la aprobación de la Agenda 2030 y los ODS por Naciones Unidas, y cuando queda menos de una década para su implementación en 2030, todavía son escasas las iniciativas implementadas en esta materia en el ámbito universitario, que se dirijan a su inclusión como un elemento de conocimiento más dentro de los programas de estudios en los grados universitarios, si bien es de justicia reconocer las iniciativas que se vienen implementando desde la Conferencia de Rectores de Universidades Españolas en los últimos años.

Segunda. En este sentido, consideramos que el presente proyecto constituye un ejemplo de buena práctica debido a su singularidad y carácter transversal, lo que lo convierte en cierto sentido en pionero a pesar de las limitaciones en su muestra y ámbito de aplicación. Este proyecto de innovación, a diferencia de otras iniciativas implementadas, destaca por ir más allá de la mera sensibilización puntual y circunscrita al ámbito de una única asignatura. Por el contrario, apuesta por una integración efectiva y global de los ODS como herramienta de aprendizaje, utilizando diferentes metodologías educativas con un carácter transversal en diferentes grados. Estas peculiaridades ponen de manifiesto, sin duda, la viabilidad de la inclusión de los ODS como un contenido más dentro de los estudios de cualquier grado universitario.

Tercera. La inclusión de los ODS en los contenidos de los estudios de los grados académicos constituye una herramienta esencial, puesto que nuestros estudiantes universitarios tendrán un papel fundamental como futuros líderes y gestores en la próxima década. A este respecto, al igual que ocurriera con los Objetivos de Desarrollo del Milenio, son numerosas las iniciativas que se están desarrollando en el ámbito de la educación primaria y secundaria auspiciadas por instituciones como la UNESCO, pero 
resultan más escasas, fragmentadas y dispersas las llevadas a cabo en el ámbito universitario, con la relevancia crucial y transformadora que las mismas pueden tener en un futuro inmediato.

Cuarta. Los resultados obtenidos tras la ejecución del proyecto constatan que los estudiantes participantes en el mismo, inicialmente, en su mayoría, tenían un casi total desconocimiento de los ODS, una situación que cambió de forma significativa tras la realización del proyecto. En algunos casos, la percepción de los estudiantes era de perplejidad, al no lograr entender cómo hasta el momento no habían tenido conocimiento de una cuestión que consideraban tan relevante. Además, como ponen de manifiesto los resultados de las encuestas realizadas, tuvo un potencial transformador en relación tanto con los contenidos académicos del grado, como en su responsabilidad y sensibilidad social. De ahí que se pueda destacar que los estudiantes otorgaran una muy buena valoración a su participación en el proyecto, 4,47 sobre un máximo de 5 y recomendaran su continuidad e incluso su extensión a otras asignaturas del grado.

Quinta. Por otra parte, la propia naturaleza del proyecto incluido dentro de la Investigación-Acción, permitió trabajar sus contenidos desde una triple dimensión, la investigadora, la académica, y la aplicada a la práctica a través de la acción. En este sentido destaca, no sólo su planteamiento de los ODS como un elemento vertebrador de cada una de las asignaturas implicadas, partiendo de un enfoque holístico y global, sino también su carácter transversal e interdependiente, que se veía complementado con una serie de actividades conjuntas que trascendían las paredes de las aulas e invitaban a los estudiantes a la acción y al compromiso social a través del aprendizaje servicio. Una dinámica de ejecución que permitió, como resultado, que los estudiantes no sólo adquirieran unos sólidos conocimientos sobre los ODS, sino además una importante educación en valores que pudieran aplicar de forma práctica en la realidad y en soft skills tan relevantes y demandados en la actualidad.

Sexta. La introducción de un variado tipo de metodologías docentes, como el aprendizaje basado en proyectos, el aprendizaje servicio, la flipped classroom, o la simulación, junto con las nuevas tecnologías, posibilitó un aprendizaje más activo y participativo por parte de los estudiantes, aunque también más trabajo y compromiso, tal y como reflejan los resultados de las encuestas. A pesar de ello, los estudiantes valoraron muy positivamente 
convertirse en protagonistas de su propio aprendizaje, y además tuvieron la oportunidad de ver de primera mano cómo, con sus pequeñas acciones, producían un impacto directo en la sociedad.

Séptima. Uno de los resultados inesperados del proyecto fue su efecto multiplicador. Así, los estudiantes implicados, tras tener conocimiento de los ODS y despertar su entusiasmo, se convirtieron en transmisores y embajadores de la Agenda 2030 entre sus amigos, conocidos y familiares, generando así un efecto multiplicador que no se había previsto inicialmente entre los resultados esperados del proyecto, lo que sin duda nos generó una grata sorpresa.

Octava. Una acción que, sin duda, revela la importancia de la función social y el compromiso que las universidades, como centros generadores de conocimiento y transmisión de valores, tienen que mantener con su entorno y con la realidad que les rodea, al tiempo que deben ser conscientes de su influencia y transformación en aras de la consecución de relevantes mejoras en beneficio de toda la sociedad.

Novena. Un proyecto que, en definitiva, aspira a poner de manifiesto cómo, ante el nuevo paradigma de acción global que nos plantean la Agenda 2030, resulta necesario que la Universidad no se quede esta vez atrás, y sea el motor de este cambio sistémico tanto a nivel educativo como en el de gestión. Comprometidos con una labor social, la Universidad debería fomentar este tipo de actividades experienciales entre nuestro alumnado, de manera transversal, como una forma de aprendizaje basado en la experiencia, además de ser una práctica que a seguir por otras universidades.

\section{Bibliografía}

APARICIO, L. y DE PAREDES, C. (2018). "Las Universidades Valencianas y los ODS", II Congreso Internacional sobre Derechos Humanos, Valencia 25 y 26 octubre 2018, Fundación Mainel.

APARICIO, L., DE PAREDES, C., ESCAMILLA, C. y GIMÉNEZ-FITA, E. (2019). Simposio: "La introducción de los ODS en las aulas universitarias". Foro Internacional 
sobre la Evaluación de la Calidad de la Investigación y de la Educación Superior (FECIES). Santiago de Compostela.

CRUE (2018). Higher Education and the 2030 Agenda: Moving into the 'Decade of Action and Delivery for the SDGs'. Crue Universidades Españolas.

CRUE (2019). Comisión de CRUE Universidades Españolas para la Agenda 2030. Crue Universidades Españolas.

DE LA ROSA, D., GIMÉNEZ, P. y DE LA CAL, C. (2019). Educación para el desarrollo sostenible: el papel de la universidad en la Agenda 2030. Revista Prisma Social, no 25.

Gobierno de España (2018). Plan de Acción para la implementación de la Agenda 2030. Hacia una Estrategia Española de Desarrollo Sostenible. Dirección General de Políticas de Desarrollo Sostenible.

ONU (2015), Resolución A/RES/70/1 "Transformar nuestro mundo: la Agenda 2030 para el Desarrollo Sostenible" aprobada por la Asamblea General el 25 de septiembre.

SACHS, JD. (2015). Achieving the sustainable development goals. Journal of International Business Ethics, vol. 8, no. 2, pp. 53-62.

Universidad Europea de Valencia (2019). I Congreso sobre Objetivos de Desarrollo Sostenible. Universidad Europea de Valencia. Valencia. https://universidadeuropea.com/valencia/noticias/la-universidad-europea-de-valenciacelebra-el-i-congreso-sobre-objetivos-de-desarrollo-sostenible 